\title{
Relações comportamentais como objeto da Psicologia: algumas implicações ${ }^{1}$
}

\author{
Emmanuel Zagury Tourinho \\ Universidade Federal do Pará
}

\begin{abstract}
RESUMO
O artigo apresenta a análise do comportamento como um sistema psicológico que assume as relações indivíduo-ambiente como seu objeto de estudos. Em seguida, discute algumas implicações teóricas, metodológicas e práticas de tal noção. A ênfase em relações comportamentais é interpretada como um contraponto a concepções individualistas do homem e a conceitos psicológicos daí derivados. Argumenta-se, por outro lado, que uma adequada apreciação do alcance da análise do comportamento como disciplina psicológica requer uma compreensão de como os conceitos analíticocomportamentais dão suporte ao exame de fenômenos psicológicos em contextos diversos daquele de laboratório, no qual a investigação básica se realiza.
\end{abstract}

Palavras-chave: análise do comportamento; behaviorismo radical; relações comportamentais.

\begin{abstract}
Behavioral relations as the subject matter of Psychology: some remarks

The paper presents behavior analysis as a psychological system that assumes individual-environment relations as its subject matter, and then discusses some theoretical, methodological, and practical implications of such a view. The emphasis on behavioral relations is interpreted as a counterpart to individualistic conceptions of man and to derived psychological concepts. It is argued, however, that an appropriate estimation of the breadth of behavior analysis as a psychological discipline requires understanding how behavior-analytic concepts support the exam of psychological phenomena in contexts that do not match those found in laboratories, where basic research is held.
\end{abstract}

Keywords: behavior analysis; radical behaviorism; behavioral relations.

O presente artigo focaliza o sistema psicológico contemporaneamente designado como "análise do comportamento", iniciando com algumas considerações sobre a definição do campo analítico-comportamental. Embora a análise do comportamento seja freqüentemente identificada com a investigação experimental de processos comportamentais básicos, o artigo enfatiza seu caráter multidimensional. Em seguida, são discutidos, à luz de proposições da análise do comportamento, um conjunto de tópicos pertinentes à reflexão sobre as fronteiras da Psicologia como um campo reflexivo, uma disciplina científica e uma profissão de ajuda.

\section{I - A análise do comportamento como sistema psicológico}

A análise do comportamento pode ser considerada um sistema psicológico porque busca prover respostas para demandas culturais dirigidas historicamente à psicologia ${ }^{2}$. De uma perspectiva histórica da psicologia, esse conjunto de demandas que dão origem à disciplina psicológica surge com a emergência e refinamento de processos de individulização, aos quais se relacionam, entre outros, uma diferenciação crescente das funções sociais (as especializações das funções), uma dissociação dos interesses e necessidades de indivíduos e grupos, a separação entre vida pública e vida privada, as práticas de auto-observação e autocontrole, as demandas por uma "eficiência produtiva" e as concepções de autonomia que acompanham as novas conformações que as relações interpessoais vão 
assumindo. Olhando muito brevemente para essas demandas e seu impacto na Psicologia, pode-se dizer que elas conduzem a (a) uma reflexão sobre o homem, sua natureza "subjetiva" e suas possibilidades de realização; (b) uma investigação científica com vistas à especificação de possíveis regularidades dos fenômenos tidos como "psicológicos"; e ao (c) desenvolvimento de estratégias, instrumentos e técnicas de ajuda, com vistas à solução de problemas que se instituem com aquele processo de individualização (cf. Tourinho, Carvalho Neto \& Neno, 2004).

$\mathrm{Na}$ medida em que o campo da psicologia vai se definindo à luz de práticas e valores forjados nos processos de individualização, ele estará fortemente regulado por uma visão de homem enquanto indivíduo, uma unidade em si mesmo, que busca (ou acredita poder) realizar-se fora do domínio das relações interpessoais. Muitas vezes a Psicologia fará a crítica dessa visão de homem. Mas talvez se possa afirmar que, mesmo quando a Psicologia se vê fazendo a crítica do individualismo, ela não institui necessariamente o terreno das relações interpessoais como o domínio de seu objeto; ela muitas vezes continua com unidades de análise que estão centradas no que ocorre com ou no indivíduo.

Respondendo às demandas que dão origem à Psicologia, a análise do comportamento se elabora como um sistema que integra produções de três tipos: (a) uma reflexão filosófica ou conceitual sobre o objeto da psicologia, as possibilidades de investigá-lo e o alcance de um conhecimento a seu respeito; (b) uma especificação de regularidades das relações comportamentais, a partir do desenvolvimento de um programa de investigações empírico-experimentais dos processos de aprendizagem; e (c) o desenvolvimento de técnicas ou estratégias de intervenção frente aos problemas humanos definidos como psicológicos (cf. Tourinho, 2003). Esses empreendimentos são por vezes designados como behaviorismo radical (a filosofia), análise experimental do comportamento (a ciência) e a prestação de serviços analítico-comportamentais. Há ainda, a análise do comportamento aplicada, em um espaço intermediário entre a investigação básica e a intervenção frente a problemas específicos. Mais no terreno das produções reflexivas, há, também, os estudos históricos em análise do comportamento, com o fim de promover uma reflexão crítica acerca do próprio desenvolvimento do sistema.

É notório que a Análise do Comportamento desenvolve-se de um modo desequilibrado do ponto de vista dos três conjuntos de produções requeridos para uma disciplina psicológica. Historicamente, seus esforços têm sido muito mais dirigidos à pesquisa científica dos processos de aprendizagem, do que à elaboração filosófica e conceitual, ou ao desenvolvimento de uma tecnologia para a intervenção. Ainda assim, merece registro o fato de que se trata de um dos poucos esforços em Psicologia com iniciativas nas três direções e, mais do que isso, um sistema que integra profissionais dedicados regularmente à produção naqueles três domínios. Isso se reflete, por exemplo, no leque de periódicos publicados na área e na diversidade da programação de seus congressos.

Recentemente, o caráter multidimensional da análise do comportamento tornou-se um tema mais freqüente no debate acerca do alcance da disciplina (cf. Donahoe, 2004; Hawkins \& Anderson, 2002; Moore \& Cooper, 2003; Rutherford, 2004; Tourinho, 1999, 2003). Essa característica vem sendo enfatizada por diversos autores, com o objetivo de colocar em discussão, entre outros, as direções para o desenvolvimento da disciplina, as estratégias de formação necessárias e a diversidade metodológica requerida. Isso sugere que uma atenção maior à necessária diversidade das produções em análise do comportamento enquanto disciplina psicológica está agora encontrando maior reconhecimento.

Mas o desenvolvimento da análise do comportamento como sistema psicológico que integra produções tão diversas não se faz em consonância com as crenças e valores que estão na origem da Psicologia. Em outras palavras, a análise do comportamento encontra-se entre aquelas abordagens que se pretendem psicológicas, ao mesmo tempo em que recusam os postulados de uma concepção individualista e subjetivista do homem. Frente às dicotomias psicológicas clássicas, o público versus o privado, o interno versus o externo, o físico versus o mental, e o objetivo versus o subjetivo, dependentes antes de tudo de uma noção mais básica de afastamento entre indivíduo e sociedade, um analista do comportamento não tomará partido por nenhuma das referências. No lugar disso, lembrará que essas categorizações não se aplicam ao seu objeto: as relações organismo-ambiente, ou mais especificamente, as relações homem-ambiente. Usando uma expressão do filósofo Gilbert Ryle (1900-1976), um analista do comportamento diria que se trata de um erro de categoria (cf. Ryle, 1949/1984) a atribuição de uma natureza mental, subjetiva, interna, ou privada a fenômenos que não são ocorrências do/no indivíduo (o conceito de indivíduo é indispensável para operar com aquelas categorias), mas relações do homem com o mundo (algumas contribuições de Ryle, 1949/1984, para uma crítica behaviorista ao mentalismo são discutidas por Baum, 1999). 
A perspectiva relacional de interpretação dos fenômenos psicológicos terá vários desdobramentos no desenvolvimento da análise do comportamento, circunscrevendo seu alcance enquanto sistema psicológico. Neste ponto, pode-se sugerir que é mais essa perspectiva relacional que limita o alcance de um projeto de psicologia como ciência do comportamento. Mais essa perspectiva, e menos um desenvolvimento desequilibrado da análise do comportamento, com sua ênfase na investigação científica.

\section{II - Implicações de uma abordagem relacional para a definição das fronteiras da Psicologia}

A perspectiva de olhar para os fenômenos psicológicos como relações do homem com o mundo - físico e social - a sua volta conduz a um conjunto amplo de decisões sobre as propriedades relevantes dos fenômenos humanos, as investigações que podem conduzir à identificação de regularidades desses fenômenos e as intervenções possíveis frente aos mesmos. Alguns exemplos dessas implicações são apresentados a seguir, com o intuito de conferir maior visibilidade aos contornos que a Psicologia vai assumindo enquanto uma disciplina comportamental. Três tópicos são brevemente considerados: a definição do objeto da Psicologia, as soluções metodológicas, e o exercício da profissão de Psicólogo.

\section{1 - Algumas implicações para a definição do objeto da Psicologia}

Relações comportamentais constituem o objeto da Psicologia, segundo a Análise do Comportamento. A terminologia empregada no tratamento dessas relações é estranha ao vocabulário mentalista que domina a comunicação cotidiana na cultura ocidental e, por vezes, estranha demais para tornar possível o diálogo com outras disciplinas ou abordagens psicológicas. Apesar disso, o núcleo da argumentação analíticocomportamental não requer uma familiaridade avançada com a abordagem para ser compreendido.

Relações comportamentais significam relações entre ações do homem e eventos do mundo físico e social com o qual ele interage. Um analista do comportamento afirma que essas ações e eventos são assumidos como constitutivos de relações apenas quando é possível especificar a função que desempenham em relação uns aos outros. Assim, quando um analista do comportamento afirma que seu objeto de estudos é o comportamento, está se referindo a uma relação funcional entre ações e eventos do mundo físico e social, ou, na sua terminologia, a uma relação funcional entre respostas e estímulos.

Levando em conta que os problemas colocados à Psicologia têm origem com o processo de individualização, é mais provável que um psicólogo enfatize as relações do homem com o mundo social, isto é, as relações interpessoais. Um analista do comportamento tenderá a aceitar essa valorização das relações interpessoais, mas chamará a atenção para o fato de que eventos do mundo físico também assumem funções importantes, mesmo quando um indivíduo não se dá conta disso. De modo mais preciso, ao analisar as relações comportamentais, será necessário destacar que o ambiente que tem uma função para o comportamento humano pode ser físico ou social, assim como pode ser histórico ou contemporâneo, público ou privado, conhecido ou desconhecido (cf. Micheletto, 2000; Skinner, 1969; Tourinho, 1997). Em outras palavras, há relações do homem com o mundo, mesmo quando os eventos do mundo que o afetam não estão presentes, não são identificados, ou não são do seu conhecimento.

Um analista do comportamento também dirá que as relações comportamentais são relações entre classes de estímulos e classes de respostas, definidas por suas funções, e não por eventuais propriedades formais, o que torna seu objeto ainda mais "fluido e evanescente", para usar expressões de Skinner (1953/1965). Ações que de um ponto de vista físico (topográfico) são muito diferentes podem ter uma mesma função no contexto de determinadas relações (compondo uma mesma classe de respostas), assim como uma mesma ação pode ter funções diversas em contextos diferentes (compondo classes de respostas diferentes). Recusar convites para eventos sociais, por exemplo, pode ter tanto a função de promover maior contato com o ambiente familiar, como de evitar cobranças sobre a aparência ou estado de humor. Nesse caso, a topografia é a mesma, mas as respostas são instâncias de classes comportamentais diferentes. Outras respostas topograficamente diferentes (por exemplo, telefonar para a família ao longo do dia) podem ter a mesma função da resposta de recusar convites. A noção de classe baseada nas funções de respostas é razoavelmente elementar no sistema explicativo skinneriano, mas merece ser ressaltada para chamar a atenção para o fato de que em sociedades complexas as topografias comportamentais podem revelar uma diversidade maior de funções, assim como também há maior diversidade topográfica, como resultado da multiplicação dos contextos com os quais os indivíduos interagem. Esse fato amplia substancialmente o escopo da análise comportamental do comportamento humano. 
As noções de classe e de função também ilustram a incompatibilidade da análise do comportamento com perspectivas fisicalistas e/ou empiricistas na definição do objeto da Psicologia, assim como sugerem a inadequação de quaisquer descrições que pretendam tratar o que pertence ao domínio das relações como substâncias ou qualidades contidas em um indivíduo. É importante assinalar que, aqui, são refutadas a um mesmo tempo as versões mentalistas e organicistas acerca dos fenômenos psicológicos. Tratar, por exemplo, o "fantasiar" ou a "lembrar" como substâncias ou ocorrências nos indivíduos, sejam essas substâncias ou ocorrências neurais ou de outra ordem, está fora de questão para uma abordagem que pretende interpretar esses fenômenos como relações do homem com o mundo.

Do ponto de vista de uma funcionalidade psicológica, classes de estímulos e classes de respostas definem-se apenas no contexto de relações; são, portanto, interdependentes. Não existe um mundo que de modo independente cause o comportamento humano, como visões mecanicistas fazem supor, assim como não existem ações humanas independentemente de uma relação com o ambiente. Para a Análise do Comportamento, o mundo que tem função para o comportamento é principalmente o mundo que é produto da ação humana, e as funções das ações humanas realizam-se apenas no contexto das relações com o ambiente. Essa perspectiva é incompatível com visões do homem como um ser passivo, que simplesmente responde ao ambiente, às vezes erroneamente atribuídas à análise do comportamento. Mas é também incompatível com visões do homem que explicam suas ações sem conectá-las com o que se passa no mundo à sua volta.

Para além disso, na análise do comportamento humano, reconhece-se que as relações comportamentais podem e freqüentemente adquirem um elevado grau de complexidade. Considerando novamente ao processo de individualização que dá origem aos problemas da Psicologia, é notório que à medida em que as relações interpessoais vão se tornando mais complexas, os fenômenos de que os psicólogos se ocupam vão adquirindo novos contornos. $\mathrm{Na}$ análise do comportamento, um modo de indicar essa complexidade consiste em assinalar que muitos fenômenos designados como psicológicos não constituem relações específicas entre respostas e estímulos, mas conjuntos de várias relações entrelaçadas, com componentes filogenéticos, ontogenéticos e culturais. Abordar na prática esses fenômenos complexos constituirá um desafio para qualquer psicólogo. $\mathrm{Na}$ investigação básica, os processos são simplificados, com o intuito de possibilitar a identificação de algumas de suas propriedades. A especificação dessas propriedades não significará, porém, que se dispõe de uma explicação suficiente para os fenômenos humanos que se apresentam nas situações concretas com as quais o profissional da Psicologia tem que lidar.

Por último, a perspectiva de interpretar os fenômenos psicológicos como relações indivíduo-ambiente é conciliada, na análise do comportamento, com o reconhecimento de que sob condições culturais que promovem uma crescente auto-observação e autocontrole, ocorrências do/no próprio indivíduo tornam-se especialmente relevantes. Não há aqui espaço para explicar como essas ocorrências são interpretadas e acomodadas em um sistema explicativo relacional (algumas indicações são encontradas em Anderson, Hawkins, Freeman \& Scotti, 2000; Anderson, Hawkins \& Scotti, 1997; Skinner, 1945, 1969, 1974/1993; Tourinho, 1995, 2006a, 2006b). Mas é indispensável registrar que a tentativa de tratar esse problema de um modo consistente é o que principalmente diferencia a análise do comportamento de outras psicologias comportamentais elaboradas ao longo do século XX.

\section{2 - Algumas implicações metodológicas}

$\mathrm{Na}$ investigação científica de relações comportamentais, o método por excelência, para a análise do comportamento, é o método experimental. Nem toda psicologia experimental, porém, é compatível com concepções analítico-comportamentais acerca dos fenômenos psicológicos; e nem só pela via experimental se chega a enunciados válidos sobre relações comportamentais.

Skinner (1945) desde cedo sugere que a análise do comportamento deve se valer também da interpretação como método. Essa possibilidade é pensada como solução para o tratamento daqueles fenômenos com alto grau de complexidade, que não se mostram acessíveis à investigação experimental. A interpretação, porém, está aqui subordinada ao arcabouço conceitual construído com o suporte da investigação empíricoexperimental. Eventos complexos podem ser abordados interpretativamente tanto quanto sejam vistos como relações comportamentais e descritos com os conceitos validados na investigação empírica de fenômenos menos complexos. Não são consideradas válidas aquelas interpretações que, conflitando com a investigação das relações comportamentais, restauram entidades do/no indivíduo como unidade de análise ou 
como descrição ou explicação do comportamento. Note-se, novamente, que é o compromisso com uma perspectiva relacional, ainda que sustentada empiricamente, que define o que vale como recurso para investigar-se os fenômenos psicológicos.

Em discussões mais recentes acerca da adoção da experimentação e da interpretação como métodos, tem sido apontado que a proposição de Skinner corresponde a definir a análise do comportamento como "dois empreendimentos interrelacionados - a análise experimental e a interpretação" (Donahoe, 2004). Donahoe prossegue, afirmando que

a análise experimental está restrita àquelas situações nas quais todas as variáveis comportamentalmente relevantes são manipuláveis, controláveis, ou mensuráveis. A interpretação é a explicação do comportamento que ocorre em situações que não atendem os requisitos da análise experimental. Essas explicações devem remeter-se exclusivamente a relações funcionais identificadas previamente em análises experimentais se é para valerem como interpretações. (p. 1)

Em uma outra direção, tem sido sugerido que um psicólogo, ao intervir frente a problemas concretos, não pode se valer de prescrições experimentais. No lugar de se ocuparem com medidas precisas dos fenômenos comportamentais, os prestadores de serviços analítico-comportamentais "precisam solucionar problemas eficientemente, de forma direta e a um custo razoável em termos financeiros e de tempo" (Moore \& Cooper, 2003, p. 82). Quando se consideram todas as condições (de pesquisa e intervenção) sob as quais o psicólogo tem que lidar com as relações comportamentais, talvez seja mais apropriado afirmar que variam ao longo de um continuum que tem como referência a observabilidade e a controlabilidade das variáveis consideradas (cf. Moore \& Cooper, 2003; Tourinho, 2004). Uma investigação experimental está mais próxima do limite desse continuum que representa maior observabilidade e controlabilidade, mas nunca alcança de modo absoluto essas condições. Do mesmo modo, as intervenções encontram-se em diferentes pontos desse continuum, nunca localizadas estritamente em um limite de absoluta inobservabilidade e incontrolabilidade.

Seja usando a experimentação, a interpretação, ou recursos metodológicos que estão a meio termo dessas soluções, as aproximações às relações comportamentais não podem ser orientadas por uma referência fisicalista do comportamento. Uma vez que o fenômeno de interesse constitui-se sempre de relações indivíduo- ambiente, será necessário criar medidas que constituam indicadores de variações nessas relações e não simplesmente de variações em ocorrências do próprio indivíduo (ainda que ocorrências acessíveis a tratamentos empírico-experimentais).

Também em razão de seu interesse por relações funcionais, a análise do comportamento tenderá a atribuir pouco valor a estudos correlacionais. Esses estudos podem ser importantes para conduzir à identificação de variáveis possivelmente relevantes em um dado contexto de ocorrência do comportamento, mas não descrevem relações propriamente ditas, pelo menos não aquelas relações (funcionais) de interesse para uma ciência do comportamento. A propósito dessa questão, um analista do comportamento tenderá a chamar a atenção para o fato de que muitas interpretações sobre as relações comportamentais estão apoiadas em uma atribuição de função causal a variáveis apenas correlacionadas com as ações de um indivíduo. Por exemplo, em alguns estudos correlacionais sobre eficácia de psicoterapia e drogas no tratamento da depressão, as variáveis etnicidade e gênero são referidas como variáveis das quais a resposta ao tratamento é função: "Também existem diferenças na expressão da depressão e em sua resposta ao tratamento como função de gênero" (Muñoz, Hollon, McGrath, Rehm \& Vandenbos, 1994, p. 53) e "Claramente, é necessário saber mais sobre as diferenças na utilização e resposta ao tratamento como função da etnicidade" (Muñoz e cols., p. 53).

Muitas vezes é em estudos correlacionais que estão apoiadas alegações sobre a determinação do comportamento humano por ocorrências do próprio indivíduo que se comporta, sustentando, assim, uma interpretação dos fenômenos psicológicos circunscrita ao indivíduo. Um analista do comportamento tenderá a levantar dois tipos de objeção a essas alegações: primeiro, a confusão entre correlação e causação; segundo, o modelo causal admitido (em geral, mecanicista, certamente não selecionista - sobre o selecionismo como modo causal, ver Skinner, 1981).

O processo de identificação de relações funcionais entre indivíduo e ambiente (físico e social) é muitas vezes referido como análise funcional. Todavia, acompanhando a variabilidade na complexidade das relações comportamentais e na observabilidade de estímulos e respostas, o conceito de análise funcional adquire diferentes conotações (cf. Neno, 2003) na análise do comportamento (e também fora dela - cf. Owens e Ashcroft, 1982). Em um contexto, ele significa uma investigação (experimental) sistemática de relações indivíduo-ambiente. Em outro, ele se apresenta como recurso empregado pelo profissional na 
intervenção (por exemplo, a terapia), permitindo inferências ou visões aproximativas de fenômenos relacionais complexos, a partir de informações obtidas por meio de relatos e/ou observações parciais. Em outras palavras, o analista do comportamento busca sempre análises funcionais das relações indivíduo-ambiente e os métodos que fundamentam suas descrições variam dependendo do problema para o qual se volta e das condições sob as quais realiza seu trabalho.

\section{3 - Algumas implicações para o exercício da profissão}

Se os métodos diferem à medida em que varia o fenômeno focalizado (em termos de complexidade, e/ou de acesso às variáveis relevantes, e/ou da controlabilidade dessas variáveis), é necessário reconhecer que no exercício da profissão de Psicólogo o que sustenta a abordagem das relações comportamentais é menos a aplicação de recursos metodológicos empírico-experimentais e mais a operação com os conceitos e enfoques produzidos a partir de pesquisas que empregam aqueles métodos. A intervenção propriamente dita vai requerer, ainda, um desenvolvimento de técnicas muito variadas, à luz da diversidade dos problemas produzidos pelo processo de individualização com os quais o profissional da Psicologia tem que lidar (cf. Neno, 2005).

Costuma-se dizer que os diferentes sistemas explicativos existentes na Psicologia é que dão origem à variedade de técnicas à disposição do profissional. De acordo com a presente interpretação, isso de fato ocorre, mas também para aqueles profissionais que operam com um mesmo sistema explicativo, a diversidade técnica será possível e mesmo necessária. Novamente, o que ligará essas técnicas umas às outras será o vínculo que todas mantêm com uma concepção relacional dos problemas para os quais estão voltadas. Para que um sistema explicativo se desenvolva, por exemplo, para que a análise do comportamento seja reconhecida enquanto um sistema que responde de modos cada vez mais amplos às demandas dirigidas à Psicologia, será necessário fomentar a variabilidade no desenvolvimento técnico. Note-se, no entanto, que se trata de uma variabilidade limitada por uma mesma perspectiva interpretativa dos fenômenos humanos.

A intervenção pautada por uma concepção relacional dos problemas psicológicos não tem como foco o que se passa com o (ou no) indivíduo. Ao abordar dimensões de sua relação com o mundo, especialmente de suas relações interpessoais, inevitavelmente essa perspectiva conflitará com uma auto-imagem individualizada. Não parece ser privilégio da análise do comportamento desfazer concepções auto-centradas do mundo; outros enfoques ou sistemas explicativos tenderão a assinalar nas relações interpessoais a gênese de vários problemas trazidos ao exame do psicólogo. Para um analista do comportamento, porém, não é suficiente afirmar que aqueles problemas têm origem em relações do homem com o mundo, para em seguida defini-los como ocorrências do/no indivíduo. E preciso ser mais radical a esse respeito e assinalar que o que é produzido naquelas relações são novos modos do homem interagir com aspectos desse mundo; os fenômenos produzidos por essas relações continuam sendo fenômenos relacionais.

Quando um analista do comportamento se define filosoficamente como behaviorista radical, o que pretende enfatizar é exatamente que o comportamento, enquanto relação do homem com o mundo, não é expressão de algo que transcende as relações; o comportamento, enquanto relação é, ele mesmo, o fenômeno psicológico.

Nas relações que os homens estabelecem com o mundo, as funções que eventos diversos assumem para cada indivíduo são idiossincráticas por força da história pessoal de cada um. Assim, não há regra para o significado que um evento qualquer pode ter para um indivíduo, e não há desvio em relação a uma natureza qualquer, quando as relações que são estabelecidas diferem substancialmente do que vem a ser $a$ norma em uma cultura.

O caráter idiossincrático das relações comportamentais que definem o repertório de um indivíduo, por um lado, obriga ao reconhecimento de uma singularidade, contrariando qualquer alegação de que a padronização é um postulado ou objetivo de uma Psicologia comportamental. Por outro, não representarão uma reintrodução dissimulada do individualismo, porque o que está sendo considerado particular não são ocorrências internas e pessoais de um indivíduo, mas suas relações com o mundo. Não são particularidades de cada um, mas particularidades de relações.

Examinando esse problema por uma outra ótica, um Psicólogo comportamental tem muito a usufruir da especificação de eventos sociais/culturais que podem ser relevantes para o comportamento dos membros de uma sociedade. A partir dessa especificação, porém, será necessário identificar as funções que esses eventos efetivamente assumem para os indivíduos. Assim, embora sejam de interesse as descrições oferecidas pelas ciências sociais para eventos sociais/culturais relevantes em um dado contexto de comportamento, só haverá uma análise propriamente psicológica quando as funções desses eventos forem estabelecidas. 


\section{CONSIDERAÇÕES FINAIS}

A análise do comportamento compartilha com algumas abordagens psicológicas e com outros sistemas explicativos das ciências humanas e sociais a crítica aos fundamentos do individualismo moderno. Apesar disso, é notória a precariedade da interlocução estabelecida com essas outras áreas da cultura que se ocupam do comportamento humano. As razões para isso são diversas e merecem ser analisadas, pois da intensificação desse diálogo depende em alguma medida um avanço na compreensão dos problemas humanos. Algumas direções para essa análise podem ser as seguintes:

a) o vocabulário da análise do comportamento não apenas é esotérico, mas conflita com o vocabulário coloquial de que fazem uso outras disciplinas. Desse ponto de vista, até mesmo frente à psicologia evolucionista, cujo programa de pesquisas parece mais claramente complementar àquele da análise do comportamento, os conflitos sobressaem-se às possibilidades de diálogo e integração (cf. Tourinho \& Carvalho Neto, 2004). Ainda que se possam salientar limites do vocabulário analítico-comportamental, porém, convém assinalar que ela evita o mentalismo ou subjetivismo que estão presentes em outros discursos e com os quais o individualismo penetra novamente na compreensão do homem oferecida pela Psicologia.

b) Dado o caráter esotérico do vocabulário analítico-comportamental muitos comentadores elaboraram visões distorcidas da abordagem, que vieram a ser amplamente disseminadas (ver, a propósito, a crítica de Chomsky, 1959). Uma atitude comum de analistas do comportamento frente à incompreensão que os críticos revelam tem sido, por vezes, simplesmente ignorar suas alegações, deixando-os sem respostas. Essa atitude tem sido menos freqüente nas últimas décadas (cf. Catania, 1991; Hernstein, 1977a, 1977b; Mahoney, 1989; Skinner, 1977), mas foi certamente suficiente em outros momentos para cristalizar em setores importantes da cultura (por exemplo, aqueles ligados à área educacional - justamente um campo da aplicação da psicologia para o qual a análise do comportamento mais se voltou) visões incorretas dos princípios e do alcance da análise do comportamento.

c) O desequilíbrio da produção analítico-comportamental também limita a criação de áreas de interlocução com outras produções culturais. Para o conjunto dos sistemas psicológicos, esse desequilíbrio constitui uma regra, mas no caso da análise do comportamento, o favorecimento da investigação científica dos processos básicos parece obscurecer o alcance da produção filosófica/conceitual que pode favorecer a interlocução, especialmente com disciplinas afins à Psicologia. O crescimento dessa produção reflexiva está em curso e poderá mudar um pouco esse cenário, talvez levando à revisão da idéia de que "o behaviorismo está morto", e permitindo a outros sistemas usufruir das importantes contribuições de uma Psicologia comportamental para a elaboração de uma concepção não individualista e não subjetivista do homem.

\section{REFERÊNCIAS}

Anderson, C. M., Hawkins, R. P., Freeman, K. A. \& Scotti, J. R. (2000). Private events: Do they belong in a science of human behavior? The Behavior Analyst, 23, 1-10.

Anderson, C. M., Hawkins, R. P. \& Scotti, J. R. (1997). Private events in behavior analysis: Conceptual basis and clinical relevance. Behavior Therapy, 28, 157-179.

Baum, W. M. (1999). Para compreender o behaviorismo: Ciência, comportamento e cultura. Porto Alegre: Artes Médicas. Tradução de M. T. A. Silva, M. A. Matos, E. Z. Tourinho e G. Y. Tomanari.

Catania, A. C. (1991). The gifts of culture and of eloquence: An open letter to Michael J. Mahoney in reply to his article, "Scientific psychology and radical behaviorism". The Behavior Analyst, 14, 61-72.

Chomsky, N. (1959). A review of B. F. Skinner's Verbal Behavior. Language, 35, 26-58.

Donahoe, J. (2004). Experimental analysis versus interpretation: An under-appreciated distinction. Resumo de trabalho apresentado na $30^{\mathrm{a}}$ Convenção da Association for Behavior Analysis, Boston. Disponível no World Wide Web: $w w w$. abainternational. org.

Hawkins, R. P. \& Anderson, C. M. (2002). On the distinction between science and practice: A reply to Thyer and Adkins. The Behavior Analyst, 25, 115-119.

Hernstein, R. J. (1977a). Doing what comes naturally: A reply to Professor Skinner. American Psychologist, 32, 1013-1016.

Hernstein, R. J. (1977b). The evolution of behaviorism. American Psychologist, 32, 593-603.

Mahoney, M. J. (1989). Scientific psychology and radical behaviorism: Important distinctions based in scientism and objectivism. American Psychologist, 44, 1372-1377.

Micheletto, N. (2000). Bases filosóficas da noção de relação funcional. Revista Brasileira de Terapia Comportamental $e$ Cognitiva, 2(2), 115-121.

Moore, J. \& Cooper, J. O. (2003). Some proposed relations among the domains of behavior analysis. The Behavior Analyst, 26, 69-84. 
Munõz, R. F., Hollon, S. D., Mcgrath, E., Rehm, L. P. \& Vandenbos, G. R. (1994). On the AHCPR depression in primary catre guidelines: Further considerations for practilioners. American Psychologist, 49, 42-61.

Neno, S. (2003). Análise funcional: Definição e aplicação na terapia analítico-comportamental. Revista Brasileira de Terapia Comportamental e Cognitiva, 5(2), 151-165.

Neno, S. (2005). Tratamento padronizado: Condicionantes históricos, status contemporâneo e (in)compatibilidade com a terapia analítico-comportamental. Tese de Doutorado. Belém: Programa de Pós-Graduação em Teoria e Pesquisa do Comportamento, Universidade Federal do Pará.

Owens, R. G. \& Ashcroft, J. B. (1982). Functional analysis in applied psychology. British Journal of Clinical Psychology, 21, 181-189.

Rutherford, A. (2004). Discussant comments. Trabalho apresentado na $30^{\mathrm{a}}$ Convenção da Association for Behavior Analysis, Boston.

Ryle, G. (1984). The concept of mind. Chicago: The University of Chicago Press. Publicado originalmente em 1949.

Skinner, B. F. (1945). The operational analysis of psychological terms. Psychological Review, 52, 270-277/291-294.

Skinner, B. F. (1965). Science and human behavior. New York/London: Free Press/Collier MacMillan. Publicado originalmente em 1953.

Skinner, B. F. (1969). Contingencies of reinforcement: A theoretical analysis. New York: Appleton-Century-Crofts.

Skinner, B. F. (1977). Hernstein and the evolution of behaviorism. American Psychologist, 32, 1006-1012.

Skinner, B. F. (1981). Selection by consequences. Science, 213, 501-504.

Skinner, B. F. (1993). About behaviorism. London: Penguin. Publicado originalmente em 1974.

Tourinho, E. Z. (1995) O autoconhecimento na psicologia comportamental de B. F. Skinner. Belém: Editora da Universidade Federal do Pará. Coleção Carlos Alberto Nunes.
Tourinho, E. Z. (1997). Privacidade, comportamento e o conceito de ambiente interno. Em R. A. Banaco (Org.), Sobre comportamento e cognição - Volume 1 (pp. 217-229). São Paulo/Santo André: ABPMC/ARBytes.

Tourinho, E. Z. (1999). Estudos conceituais na análise do comportamento. Temas em Psicologia da SBP, 7, 213-222.

Tourinho, E. Z. (2003). A produção de conhecimento em psicologia: A análise do comportamento. Psicologia: Ciência e Profissão, 23(2), 30-41.

Tourinho, E. Z. (2004). Definições contemporâneas da análise do comportamento. Trabalho apresentado na XXXIV Reunião Anual da Sociedade Brasileira de Psicologia, Ribeirão Preto/SP.

Tourinho, E. Z. (2006a). Private stimuli, covert responses and private events: Conceptual remarks. The Behavior Analyst, 29(1), 13-31.

Tourinho, E. Z. (2006b). Subjetividade e relações comportamentais. Tese (Professor Titular). Belém: Universidade Federal do Pará: Departamento de Psicologia Experimental.

Tourinho, E. Z. \& Carvalho Neto, M. B. (2004). O conceito de estado inicial na explicação do comportamento humano: Considerações de uma perspectiva analítico-comportamental. Em Moura, M. L. S. (Org.), $O$ bebê no século XXI e a psicologia em desenvolvimento (pp. 111-134). São Paulo: Casa do Psicólogo.

Tourinho, E. Z., Carvalho Neto, M. B. \& Neno, S. (2004). A psicologia como campo de conhecimento e como profissão de ajuda. Estudos de Psicologia (Natal), 9, 17-24.

Recebido: $15 / 05 / 2006$ Revisado: 15/06/2006 Aceito: $21 / 06 / 2006$

\section{Notas:}

${ }^{1}$ Trabalho parcialmente financiado pelo Conselho Nacional de Desenvolvimento Científico e Tecnológico (Processos 305743/2004-0 e 470802/2004-9). Uma versão preliminar foi apresentada no IV Congresso Norte-Nordeste de Psicologia, Salvador, maio de 2005.

${ }^{2}$ Desse ponto de vista, a análise do comportamento não se constitui simplesmente como um ramo da biologia (Skinner, 1969), ainda que esse tipo de referência seja compreensível à luz de sua ênfase no fato de que está lidando com fenômenos naturais (no sentido de não transcendentais) dos organismos.

\section{Sobre o autor:}

Emmanuel Zagury Tourinho: Doutor em Psicologia Experimental pela Universidade de São Paulo. Professor adjunto na Universidade Federal do Pará. Endereço eletrônico: tourinho@amazon. com. br. 